Commun. math. Phys. 37, 141-160 (1974)

(C) by Springer-Verlag 1973

\title{
On the Local Central Limit Theorem for Gibbs Processes
}

\author{
G. Del Grosso \\ Istituto Matematico, Università di Roma, Roma, Italy \\ Received April 10, 1973; in revised form January 14, 1974
}

\begin{abstract}
We derive a sufficient condition for the validity of the local central limit theorem for Gibbs processes and their isomorphism with a Bernoulli shift.
\end{abstract}

\section{Introduction}

It has been recently realized to the class of $\delta$-dimensional discrete time stationary Markov processes and the class of translation invariant finite range Gibbs processes are in one-to-one correspondence and define the same class of random fields [1-3] (see below for a precise statement).

To fix the notations we briefly recall the definitions. For simplicity the space of states will be restricted to be $I=[0,1]$ : the generalization to $I$ finite is straightforward.

Definition 1. A stationary Markov process on a $\delta$-dimensional lattice $Z^{\delta}$ is

i) a translation invariant Borel probability measure $\mu$ on $I$ endowed with the product topology ( $I$ being considered with the discrete topology);

ii) $\mu$ has the property that if $\Lambda \subset Z^{\delta}$ is a finite region then the probability distribution of the events inside $\Lambda$ is independent on the events outside

$$
\Lambda_{d} \equiv\left\{\xi / \xi \in Z^{\delta} / \Lambda, d(\xi, \Lambda) \leqq d, d(\xi, \Lambda)=\text { distance of } \xi \text { from } \Lambda\right\}
$$

where $0 \leqq d<\infty$ and $d$ depends on $\mu$ but not on $\Lambda$.

Before defining a Gibbs process observe that $I^{Z^{\hat{\delta}}}$ can be regarded as the set of subsets $X \subset Z^{\delta}$. If $X \in I^{Z^{\delta}}$ is regarded as a subset of $Z^{\delta}$ we shall call its points the "occupied points" and we shall refer to $X$ as to a "configuration" 1 .

${ }^{1}$ It would be more appropriate to call such a process a $d$-Markov process $I$ or a finite memory process since, if $\delta=1$, it does not reduce to the equal definition of Markov process (unless $d=1$ ). However it is known that one-dimensional finite memory processes are Markov processes on a different space of states (see, for istance, [1]). 
Definition 2. A translationally invariant Gibbs process on $Z^{\delta}$ is

i) a translation invariant Borel probability measure on $I^{Z^{\delta}}$;

ii) a translationally invariant (real) function $\Phi$ defined over the subsets of $Z^{\delta}$ such that

$$
\Phi(X) \equiv 0 \quad \text { if } \quad \operatorname{diam} X>d .
$$

This function should allow express the conditional probability $f(X / Y)$ that a randomly chosen element $T \in I^{Z^{\delta}}$ is such that $T \cap A=X$ under the condition that $T \cap \Lambda=Y$, this conditional probability should be given by:

$$
f_{\Lambda}(X / Y)=N(Y) \exp \cdot \sum_{\emptyset \neq S \subset X} \sum_{P \subset Y} \Phi(S \cup P)
$$

where $N(Y)$ is a normalization constant and Equality (1.1) is to be understood almost everywhere in the Kolmogorov's sense [5].

Definition 3. The function $\Phi$ above is called the potential of the process and will be thought as a pair $\Phi \equiv\left(\mu, \Phi^{\prime}\right)$ where $\mu=-\Phi(\xi)$ $\left(\xi \in Z^{\delta}\right.$ : one body or chemical potential) and $\Phi^{\prime}$ (many body component of the potential) is a new potential such that $\Phi^{\prime}(X)=\Phi(X)$ if $|X|=$ (number of points in $X)>1$ and $\Phi^{\prime}(\xi)=0$ if $\xi \in Z^{\delta}$.

Notice that the translation invariance implies that $\mu$ is a constant.

The notion of Gibbs process has been introduced in probability theory by Dobrushin [1] and in Mathematical Physics by Dobrushin [1] and, independently, Lanford, Ruelle [4]. Of course Definition 2 can be generalized to the case $d=+\infty$ : in this case, in order that (1.1) makes sense, one has to require $[1,4]$ :

$$
\|\Phi\|=\sum_{S \ni \xi}\left|\Phi^{\prime}(S)\right|<+\infty
$$

notice that $\|\Phi\|$ is $\xi$-independent for $\xi \in Z^{\delta}$.

The following theorem holds ${ }^{2}$ :

Theorem 1. There is one-to-one correspondence between non-singular (see below) stationary Markov processes and translationally invariant finite range Gibbs processes. Two corresponding processes are described by the same probability measure $\mu$. Here a non-singular Markov process is a process $\mu$ such that:

$$
\mu\left(\left\{T / T \subset Z^{\delta}: T \cap \Lambda=X\right\}\right)>0 \quad \forall \text { finite } \Lambda, \forall X \subset \Lambda .
$$

It is now relevant to ask wether or when the local central limit theorem is valid for the above processes.

2 The proof of this theorem has been published in the case $d=1,2$. It is, however, a consequence implicit in the proof of the theorem of Griffiths and Ruelle [3]. Notice that only the direct part of Theorem 1 is not trivial. The case $d=1$ has been also proved by Tesei (private communication). 
We shall put the problem in the following form: let $\Lambda \subset Z^{\delta}$ be a finite region and put, for $X \subset Z^{\delta},|X|=(\#$ points in $X)$. Consider:

$$
p(k ; \Lambda)=\mu\left(\left\{T / T \subset Z^{\delta},|T \cap \Lambda|=k\right\}\right)
$$

where $k=0,1,2, \ldots$ is an integer; i.e. $p(k ; \Lambda)$ is the probability that a randomly chosen $T \in I^{Z^{\delta}}$ contains exactly $k$ points inside $\Lambda$. Then we ask wether or when:

$$
p(k ; \Lambda)=\frac{\exp \left\{-(k-\langle k\rangle)^{2} / 2 \sigma^{2}|\Lambda|\right\}}{\sqrt{2 \pi \sigma^{2}|\Lambda|}}+O\left(\frac{1}{\sqrt{|\Lambda|}}\right)
$$

where $\langle k\rangle$ is the average value, with respect to (1.4), if the random variable $k$ and $\sigma^{2}$ is a constant dependent only on $z$ and $\|\Phi\|$.

Clearly a result of the type (1.5) is true when $\delta=1$ and $\Lambda$ is an interval as a consequence of Kolmogorov's theorem [5].

However for $\delta>1$ the statement might fail to be true even for square $\Lambda$ 's and is known to be false in some of the simplest cases (i.e. $d=1$; known as Ising model). In the known examples the faillure of the local central limit theorem is connected with what is known in physics as a phase transition (see Section 5).

In this paper we show how a powerful technique, developed in connection with problems in Statistical Mechanics, can be applied to construct a proof of a number of results which are also known to follow by the combined use of the theorems proved in $[1,7]$. We provide some sufficient criteria on $\Phi$ for the validity of (1.5). The estimates we give are certainly not the best since the aim of this paper is to describe how a technique, well known in Statistical Mechanics of low density [6], applies to our problem.

As a byproduct we also obtain that, when (1.5) holds, the Gibbs process is isomorphic, as a $\delta$-dimensional dynamical system, to a Bernoulli shift with the same entropy: this will follow by combining the formula ( ) in [15] and the formula (3.9) of Lemma 2.

It is known that similar results have been obtained in the works of Ryauba [7], Halfina [16], Minlos and Halfina [17]; our technique is, however, completely different and also allows to obtain stronger results (like the Bernoullicity of the process).

\section{The Main Results}

We shall recall that the conditional probability $f_{A}(X / Y)$ given in $(1,1)$ is linked with the measure $\mu$ on $\left(I^{2^{\delta}}, \mathfrak{U}\right)$ where $\mathfrak{U}$ is the $\sigma$-algebra generated by the subsets of the form $\left\{Y \mid Y \subset Z^{\delta}, Y \cap M=X\right\}$ for some finite $M \subset Z^{\delta}$, by the following relations: if $\Lambda$ is fixed a probability measure 
on $I^{Z^{\delta} / \Lambda}$ can be defined for every $X \subset A$, by

$$
\int F d \mu=\sum_{X \subset A} \int F(X \cup Y) \mu_{\Lambda}(X, d Y)
$$

where $F$ belongs to the $\sigma$-algebra of cilindrical functions on $I^{Z^{\delta}}$ (i.e. of continuous functions on $I^{Z^{\delta}}$ such that $F(X)=F(X \cap T)$ for every $X \in I^{Z^{\delta}}$ and some finite $\left.T \subset Z^{\delta}\right)$, therefore the conditional probability $f_{\Lambda}(X / Y)$ is given by

$$
\mu_{\Lambda}(X, d Y) \equiv f_{\Lambda}(X, d Y) Q_{\Lambda}(d Y)
$$

where $Q_{A}$ is the measure on $I^{Z^{\delta} / \Lambda}$ defined as follows

for any $E \in I^{Z^{\delta} / \Lambda}$.

$$
Q_{\Lambda}(E)=\mu\left(\left\{X \mid X \cap Z^{\delta / \Lambda} \in E\right\}\right)
$$

Let $\mu$ be now a non singular $d$-Markov process and $\Phi$ be the corresponding Gibbs potential with range $d$ (see Theorem 1).

Let $f_{A}(X / Y)$ be the conditional probability functions associated with $\mu$ [see (1.1)] and consider, for fixed $Y \subset Z^{\delta} / \Lambda$, the quantity

$$
p(k ; \Lambda / Y)=\sum_{\substack{X \subset \Lambda \\|X|=k}} f_{\Lambda}(X / Y),
$$

i.e. the conditional probability for finding exactly $k$ occupied points inside $\Lambda$ knowning that the configuration outside $\Lambda$ is $Y$.

Introduce the following notations ${ }^{3}$ :

$$
\begin{gathered}
\varepsilon_{d}(\Lambda)=|\Lambda|^{-1} \cdot(\# \text { points within } d \text { from } \partial \Lambda) \\
O(\alpha)=\vartheta \alpha \text { for } \alpha \text { real if }|\vartheta| \leqq 1 \\
C(\Phi)=2 e^{\|\Phi\|}\left(\exp \left(e^{\|\Phi\|}-1\right)-1\right)
\end{gathered}
$$

where $\vartheta$ may depend on everything possible (but $|\vartheta| \leqq 1$ ); $\|\Phi\|$ has been introduced in (1.2).

The following theorems are our main result:

Theorem 2. Let $\mu$ be a Gibbs process with range $d$ and let $\Phi=\left(\mu, \Phi^{\prime}\right)$ (see Definition 3) be its potential. Assume that:

$$
\sum_{h=2}^{\infty} \frac{5}{4}\left[e^{\mu} C(\Phi)\right]^{h-1} h^{2}<1
$$

then there are functions $\gamma=\gamma\left(e^{\mu},\|\Phi\|\right), \sigma^{2}=\sigma^{2}\left(e^{\mu}, \Phi^{\prime}\right)$ such that for all $\Lambda \subset Z^{\sigma},|\Lambda|<\infty$ and all $Y \subset Z^{\delta} / \Lambda$

$$
p(k ; \Lambda / Y)=\frac{1}{\sqrt{2 \pi \sigma^{2}|\Lambda|}} \cdot e^{-\frac{1}{2 \sigma^{2}|\Lambda|} \cdot\left(k-\langle k\rangle_{Y}\right)^{2}}+\gamma \cdot \frac{O\left(\varepsilon_{d}(\Lambda)\right)}{\sqrt{|\Lambda|}}
$$

where $\langle k\rangle$ is the average of the random variable $k$ with respect to (2.4).

\footnotetext{
${ }^{3}$ The boundary $\partial \Lambda$ of $\Lambda$ consists in the points of $\Lambda$ having a neighbor not in $\Lambda$.
} 
A consequence of the $Y$ and $\Lambda$ independence of $\sigma^{2}$ and $\gamma$ as well as (3.9), that we shall see later, will be the following local central limit theorem for the distribution (1.7):

Theorem 3. Under the same assumptions and notations of the Theorem 2 the following holds:

$$
p(k ; \Lambda)=\frac{1}{\sqrt{2 \pi \sigma^{2}|\Lambda|}} e^{-\frac{1}{2 \sigma^{2}|\Lambda|}(k-\langle k\rangle)^{2}}+\gamma \frac{O\left(\varepsilon_{d}(\Lambda)\right)}{\sqrt{|\Lambda|}}
$$

where $\langle k\rangle$ is the average of $\langle k\rangle_{Y}$ with respect to (1.4).

Notice that no assumptions are made on $\Lambda$ (except $|\Lambda|<+\infty$ ): it may be of arbitrary shape and even disconnected.

\section{Technicalities}

In this section we introduce the notations necessary to formulate Lemmas 1 and 2. These lemmas will be the main tool to be used for the proof of the Theorems 2 and 3.

Let $\Lambda$ be a finite region $\Lambda \subset Z^{\delta}$ and fix $Y \subset Z^{\delta} / \Lambda$ and let $\lambda(\xi)$ be one arbitrary complex function defined on $Z^{\delta}$.

It will prove usefull to study the quantity

where

$$
Z(\Lambda, \lambda)=\sum_{X \subset A} e^{-U(x)-I(X)} \prod_{\xi \in X}[z \lambda(\xi)]
$$

and

$$
z=\exp \mu
$$

$$
\begin{gathered}
U(X)=\sum_{\sigma \neq S \subset X} \Phi^{\prime}(S) \\
I(X)=I(X, Y)=\sum_{\substack{\sigma \neq S \subset X \\
\sigma \neq P \subset Y \\
\Phi=\left(\mu, \Phi^{\prime}\right) .}} \Phi^{\prime}(S \cup P) \\
\Phi, ~
\end{gathered}
$$

It is clear, after comparison with (1.1), that when $\lambda(\xi)=1$, the function is the normalization constant $N(Y)^{-1}$ in (1.1). Our main problem in this section is to recast (3.1) into a more handable form and this is achieved through the following Lemma 1.

We need first some notations.

Let $\mathfrak{P}(\Lambda)$ be a family of sequences $\left\{n_{\xi}\right\}$ with $\xi \in \Lambda$ and $n_{\xi}=0,1,2, \ldots$. $\mathfrak{P}\left(Z^{\delta}\right)$ will be the set of the sequences $\left\{n_{\xi}\right\}, \xi \in Z^{\delta}$ s.t. $\sum_{\xi} n_{\xi}<\infty$. Obviously we can regard the elements of $\mathfrak{B}(\Lambda)$ as subsets of $\Lambda$ with multiplicities: they will be denoted by capital letters (as before we have denoted the 
subsets of $Z$ : no confusion should however arise). If $X \in \mathfrak{P}(\Lambda), \tilde{X}$ will be denoted the set of points $\xi \in \Lambda$ such that $n_{\xi} \geqq 1$ (i.e. the set of occupied points). Furthermore we put

$$
\begin{aligned}
|X| & =(\# \text { points in } X)=\sum_{\xi} n_{\xi} \geqq|\tilde{X}| \\
X ! & =\prod_{\xi \in A} n_{\xi} ! \\
\lambda(X) & =\prod_{\xi \in X}[\lambda(\xi)]^{n_{\xi}}
\end{aligned}
$$

clearly $|X|=|\tilde{X}|$ iff $X !=1$.

The following lemma holds:

Lemma 1. If $z C(\Phi)<1$ then there is a real function $\varphi_{\Lambda}^{T}(X)$ on $\mathfrak{P}(\Lambda)$ s.t. for all $\lambda$ 's with the property $|\lambda(\xi)| \leqq 1$.

$$
Z(\Lambda, \lambda)=\exp \sum_{X \in \mathfrak{B}(\Lambda)} z^{|X|} \lambda(X) \frac{\varphi_{\Lambda}^{T}(X)}{X !} ;
$$

ii) if the distance $d(X, \partial \Lambda)$ of $X$ from $\partial \Lambda$ exceeds $d$ the functions $\varphi_{\Lambda}^{T}(X)$ are $Y$ and $\Lambda$ independent (i.e. depend only on $\left.\Phi\right)$;

iii)

$$
\sum_{\substack{X \ni \xi \\|X|=k}} \frac{\left|\varphi_{\Lambda}^{T}(X)\right|}{X !} \leqq \mathrm{C}(\Phi)^{k-1} \quad \mathrm{k}=1,2, \ldots ;
$$

iv) there are two functions $K(\alpha, z,\|\Phi\|)$ and $k(z,\|\Phi\|)$ s.t. for all real $\alpha$ 's

$$
\sum_{\substack{X \supsetneqq \xi_{,}, \xi^{\prime} \\ X \in \mathfrak{P}(\Lambda)}} \frac{\left|\varphi_{\Lambda}^{T}(X)\right|}{X !}|X|^{\alpha} z^{|X|} \leqq K(\alpha, z,\|\Phi\|) e^{-d\left(\xi, \xi^{\prime}\right) k(z,\|\Phi\|)} .
$$

The proof of this lemma is given in Appendix A: it consists essentially in a rewording of Section 6 of Ref. [8] which, however, contained some combinatorial errors (although the final results were correct).

Now if $\Lambda \subset Z^{\delta}$ is a finite region of $Z$ we define the absolute probability that the only points occupied in $\Lambda$ are the ones in $Y$, as follows

$$
f_{\Lambda}(Y)=\mu\left(\left\{T \mid T \subset Z^{\delta}: T \cap A=Y\right\}\right) .
$$

Clearly the $f_{\Lambda}(Y)$ 's functions have to satisfy the following properties of consistency

$$
\begin{array}{ll}
\text { i) } \quad \sum_{Y \subset \Lambda} f_{\Lambda}(Y)=1 ; & \\
\text { ii) } \sum_{X \subset \Lambda / T} f_{\Lambda}(X \cup Y)=f_{T}(Y) & Y \subset T \subset \Lambda \\
& X \subset \Lambda .
\end{array}
$$

It will be shown in the Appendix B the following lemma. 
Lemma 2. If $z C(\Phi)<1, \Lambda_{1}$ and $\Lambda_{2}$ are two finite regions of $Z^{\delta}$ with $d\left(\Lambda_{1}, \Lambda_{2}\right) \geqq 3 d$ and $X_{1} \subset \Lambda_{1}, X_{2} \subset \Lambda_{2}$ the following property for the $f_{\Lambda}(Y)$ 's functions holds:

$$
\left|f_{\Lambda_{1} \cup \Lambda_{2}}\left(X_{1} \cup X_{2}\right)-f_{\Lambda_{1}}\left(X_{1}\right) f_{\Lambda_{2}}\left(X_{2}\right)\right| \leqq f_{\Lambda_{1}}\left(X_{1}\right) f_{\Lambda_{2}}\left(X_{2}\right) e^{-\alpha d\left(\Lambda_{1}, \Lambda_{2}\right)}
$$

where $d\left(\Lambda_{1}, \Lambda_{2}\right)$ is the distance between $\Lambda_{1}$ and $\Lambda_{2}$ and $\alpha=\alpha(z,\|\Phi\|)$ is a constant.

Inequality (3.9) can be used to prove that the $\delta$-dimensional Markov processes are Bernoulli shifts under the assumptions of Lemma 2.

\section{Proof of the Theorem 2}

We prove the Theorem 2 following the Gnedenko's method for the proof of the local central limit theorem for independent variables [9]: therefore we write the characteristic function of random variable $k=|X|$, $X \subset \Lambda$

$$
\begin{aligned}
\psi(t) & =\sum_{k=0}^{|A|} e^{i k t} p(k ; \Lambda / Y) \\
& =\frac{\sum_{X \subset \Lambda} e^{-U(X)-I(X)} z^{|X|} e^{i, t|X|}}{\sum_{X \subset \Lambda} e^{-U(X)-I(X)}}=\frac{Z\left(\Lambda, e^{i t}\right)}{Z(\Lambda, 1)} \\
& =\exp \sum_{X \in \mathfrak{P}(\Lambda)} z^{|X|}(\lambda(X)-1) \frac{\varphi_{\Lambda}^{T}(X)}{X !}
\end{aligned}
$$

where in the last step the Lemma 1 has been applied and we have put $=e^{i t|X|}=\lambda(X)$. Therefore

$$
p(k ; \Lambda / Y)=\frac{1}{2 \pi} \int_{-\pi}^{+\pi} e^{-i t k} \psi(t) d t .
$$

We need now a simple expression for $\psi(t)$ in order to perform the integral (4.2). Develop the argument of the last exponential in (4.1) according to the Schlömilch formula of third order in $t$ ( $t$ appears in $\left.\lambda(X)=e^{i t|X|}\right)$

$$
\begin{aligned}
\sum_{X \in \mathfrak{P}(\Lambda)} z^{|X|}(\lambda(X)-1) & \frac{\varphi_{\Lambda}^{T}(X)}{X !} \\
= & i t\langle k\rangle_{Y}-\frac{t^{2}}{2} \sum_{X \in \mathfrak{P}(\Lambda)} z^{|X|}|X|^{2} \frac{\varphi_{\Lambda}^{T}(X)}{X !} \\
& +\frac{(i t)^{3}}{6} \sum_{X \in \mathfrak{P}(\Lambda)}|X|^{3} z^{|X|} e^{i \vartheta t|X|} \frac{\varphi_{\Lambda}^{T}(X)}{X !}
\end{aligned}
$$


where the identification $\langle k\rangle_{Y}=\sum_{X \in \mathfrak{P}(\Lambda)} z^{|X|}|X| \frac{\varphi_{\Lambda}^{T}(X)}{X !}$ follows, as usual, by differentiating 1.h.s. and r.h.s. of (4.1) with respect to $t$ and setting $t=0$.

We now extract the leading contribution (as $|\Lambda| \rightarrow \infty)$ to the sums $\sum_{X \in \mathfrak{P}(\Lambda)}$ in (4.3). To do so we need to use (ii) of Lemma 1: call $\varphi^{T}(X)$ the translation invariant value of $\varphi_{\Lambda}^{T}(X)$ when $d(X, \partial \Lambda)>d$ then

$$
\begin{gathered}
\sum_{X \in \mathfrak{P}(\Lambda)} z^{|X|}(\lambda(X)-1) \frac{\varphi_{\Lambda}^{T}(X)}{X !} \\
=i t\langle k\rangle_{Y}-\frac{t^{2}}{2} \sum_{\substack { \xi \in \Lambda \\
\begin{subarray}{c}{X \ni \xi \\
X \in \mathfrak{P}(\Lambda){ \xi \in \Lambda \\
\begin{subarray} { c } { X \ni \xi \\
X \in \mathfrak { P } ( \Lambda ) } }\end{subarray}} z^{|X|} \frac{|X|^{2}}{|\tilde{X}|} \frac{\varphi_{\Lambda}^{T}(X)}{X !} \\
+\frac{(i t)^{3}}{6} \sum_{\xi \in \Lambda} \sum_{\substack{X \ni \xi \\
X \in \mathfrak{P}(\Lambda)}} z^{|X|}|X|^{3} \cdot e^{i t \vartheta|X|} \frac{\varphi_{\Lambda}^{T}(X)}{X !} \\
=i t\langle k\rangle_{Y}-\frac{t^{2}}{2}|\Lambda| \sum_{\substack{X \ni 0 \\
X \in \mathfrak{P}\left(Z^{\delta}\right)}} z^{|X|} \frac{|X|^{2}}{|\tilde{X}|} \frac{\varphi^{T}(X)}{X !}+\frac{t^{2}}{2}|\Lambda| \cdot M(z,\|\Phi\|) \vartheta\left(\varepsilon_{d}(\Lambda)\right) \\
+t^{3}|\Lambda| \cdot M_{1}(z,\|\Phi\|) \vartheta\left(\varepsilon_{d}(\Lambda)\right)
\end{gathered}
$$

where in $(4.4 \mathrm{c})$ the third term of takes into account the error involved in our approximation. This error is due to two facts: if in the $t^{2}$ term of (4.4b) the summation $\sum_{\xi \in \Lambda}$ is replaced by the factor $|\Lambda|$ and $X$ runs over $\mathfrak{P}\left(Z^{\delta}\right)$, so we obtain, obviously, a larger number of terms than it was correct. Furthermore in correspondence with the $X$ 's sets such that $X \cap \Lambda_{d}^{\prime} \neq \emptyset$ (where $\Lambda_{d}^{\prime}=\{\xi \mid \xi \in \Lambda, d(\xi, \partial \Lambda) \leqq d\}, d=$ range $\left.\Phi\right)$, the true value has been replaced by the corresponding translation invariant one $\varphi^{T}(X)$.

Using ii), iii) of Lemma 1, it is easy to demonstrate that this error is bounded by a function only dependent on $\|\Phi\|$ and $z$ : this explains the fact that $M$, in the last expression of (4.4) in a function of $\|\Phi\|$ and 2 . By the same reasoning it is possible to verify that the $t^{3}$ term (of 4.4) it can be expressed in the form $t^{3}|\Lambda| M_{1}(z,\|\Phi\|) O\left(\varepsilon_{d}(\Lambda)\right)$. We shall put now

$$
\sigma^{2}=\sum_{X \ni \xi} z^{|X|} \frac{|X|^{2}}{|\tilde{X}|} \frac{\varphi^{T}(X)}{X !}
$$

and notice that (ii) Lemma 1 together with (2.5) imply

$$
\sigma^{2}>z-\sum_{h=2}^{\infty} z^{h} C(\Phi)^{h-1} h^{2}>0 .
$$


Expression (4.4) turn out be useful for small $t$; for larger $t$ 's we need only rough estimates: suppose $|\Lambda|^{-1 / 2+1 / 6} \leqq|t| \leqq \eta$ where $\eta$ will be chosen later. Then

$$
\begin{aligned}
& \left|\exp \sum_{X \in \mathfrak{P}(\Lambda)} z^{|X|}(\lambda(X)-1) \frac{\varphi_{\Lambda}^{T}(X)}{X !}\right| \\
& \quad \leqq \exp -\frac{t^{2}}{2} \sum_{X \in \mathfrak{P}(\Lambda)} z^{|X|}|X|^{2}\left(\operatorname{Re} e^{i t \vartheta|X|}\right) \frac{\varphi_{\Lambda}^{T}(X)}{X !}
\end{aligned}
$$

where we have developed the argument of the exponential with the second order Schlömilch formula in $t$.

Then

$$
\begin{aligned}
\text { (1.h.s. of } 4.6) \leqq & \exp \left\{-\frac{t^{2}}{2}|\Lambda| \sum_{\substack{X \ni \xi \\
X \in \mathfrak{B}\left(Z_{2}\right)}} z^{|X|} \frac{|X|^{2}}{|\tilde{X}|} \cos t \vartheta|X| \frac{\varphi^{T}(X)}{X !}\right. \\
& +t^{2}|\Lambda| \cdot M_{2}(z,\|\Phi\|) O\left(\varepsilon_{d}(\Lambda)\right)
\end{aligned}
$$

where the inequality has been derived from (4.6) by extracting its leading contribution with the same method used for (4.3), (4.4) and $M_{2}$ is a suitable function.

Notice that since $\varphi^{T}(X)=1$ if $|X|=1$

$$
\sum_{\substack{X \ni \xi \\ X \in \mathfrak{P}(\Lambda)}} z^{|X|} \frac{|X|^{2}}{|\tilde{X}|} \frac{\varphi^{T}(X)}{X !} \cos |t| \vartheta|X| \geqq z \cos \eta-\sum_{h=2}^{\infty} h^{2} z^{h} G(\Phi)^{h-1} .
$$

Finally suppose $\eta \leqq|t| \leqq \pi$ : we find, as for (4.4), (4.6), (4.7)

$$
\begin{aligned}
& \left|\exp \sum_{X \in \mathfrak{P}(\Lambda)} z^{|X|}\left(e^{i|t||X|}-1\right) \frac{\varphi_{\Lambda}^{T}(X)}{X !}\right| \\
& \equiv \exp -\sum_{X \in \mathfrak{P}(\Lambda)} z^{|X|}(1-\cos |t||X|) \frac{\varphi_{\Lambda}^{T}(X)}{X !} \\
& =\exp \left\{-|\Lambda| \sum_{\substack{X \ni \xi \\
X \in \mathfrak{P}(\Lambda)}} z^{|X|} \frac{(1-\cos |t||X|)}{|\tilde{X}|} \frac{\varphi^{T}(X)}{X !}+\Lambda M_{3}(z,\|\Phi\|) O\left(\varepsilon_{d}(\Lambda)\right)\right\}
\end{aligned}
$$

and notice that

$$
\sum_{\substack{X \ni \xi \\ X \in \mathfrak{P}(\Lambda)}} z^{|X|} \frac{(1-\cos |t||X|)}{|\tilde{X}|} \frac{\varphi^{T}(X)}{X !} \geqq z(1-\cos \eta)-\sum_{h=2}^{\infty} z^{h} C(\Phi)^{h-1}
$$

We now choose $\eta$ so that the 1.h.s. of (4.8), (4.10) are both positive: this is possible because of (2.5). 
It is now easy to evaluate the integral (4.2) using the Gnedenko's method. This is most conveniently done by introducing the new variable $\tau=t \sqrt{|\Lambda|}$ and by decomposing the integration interval in three parts:

$$
|\tau| \leqq|\Lambda|^{1 / 6} ; \quad|\Lambda|^{1 / 6} \leqq|\tau| \leqq \eta \sqrt{|\Lambda|} ; \quad \eta \sqrt{|\Lambda|} \leqq|\tau| \leqq \pi \sqrt{|\Lambda|} .
$$

It follows that the contributions of the last two integrals are exponentially small as $|\Lambda| \rightarrow \infty$ (i.e. they go to zero as $\exp -|\Lambda|^{\alpha}$ with $0<\alpha<1-\frac{1}{3}$ ) provided $O\left(\varepsilon_{d}(\Lambda)\right) \rightarrow 0$ (i.e. if $\Lambda$ is no too irregular), while the first region gives the main contribution.

Formula (2.9) is found performing the easy gaussian integrals and after some little algebraic transformations.

\section{Proof of the Theorem 3}

To prove Theorem 3 remark that

$$
\langle k\rangle_{Y}=\langle k\rangle+\delta\langle k\rangle_{Y}
$$

where $\langle k\rangle$ is the mean value of random variable $k$ when $Y$ is no fixed and $\langle k\rangle_{Y}$ is the mean value of $k$ with respect to (2.4).

Of course the average of $\langle k\rangle_{Y}$ over the condition $Y$ is $\langle k\rangle$ hence $\left\langle\delta\langle k\rangle_{Y}\right\rangle=0$.

If we substitute (5.1) into (2.9) expanding the exponential function by Schlömilch formula up to the 2-nd order in $\delta\langle k\rangle_{Y}$ and taking the mean value with respect to $Y$ we obtain:

$$
\begin{aligned}
& \langle p(k ; \Lambda / Y)\rangle=p(k ; \Lambda) \\
& \quad=\frac{1}{\sqrt{2 \pi \sigma^{2}|\Lambda|}}\left\{e^{-\frac{(k-\langle k\rangle)^{2}}{2 \sigma^{2}|\Lambda|}}+\frac{\left\langle\left(\delta\langle k\rangle_{Y}\right)^{2}\right\rangle}{2 \sigma^{2}|\Lambda|} O M_{4}\right\}+\gamma \frac{O\left(\varepsilon_{d}(\Lambda)\right)}{\sqrt{|\Lambda|}}
\end{aligned}
$$

where $M_{4}=\max _{-\infty \leqq r \leqq+\infty} \frac{e^{-r^{2}}\left|4 r^{2}-2\right|}{2 \sigma^{2}}$. Therefore we must now only demonstrate that $\left\langle\left(\delta\langle k\rangle_{Y}\right)^{2}\right\rangle$ is the same order as $O\left(\varepsilon_{d}(\Lambda)\right)$. Since

$$
\begin{aligned}
\left\langle\left(\delta\langle k\rangle_{Y}\right)^{2}\right\rangle= & \sum_{\substack{\xi \in A \\
\zeta \in \Lambda}} \sum_{\substack{\xi \in X \in \mathfrak{P}(\Lambda) \\
\zeta \in Z \in \mathfrak{P}(\Lambda)}} z^{|X|+|Z|} \frac{|X||Z|}{|\tilde{X}||\tilde{Z}|}\left\langle\left(\left\langle\frac{\varphi_{\Lambda}^{T}(X)}{X !}\right\rangle-\frac{\varphi_{\Lambda}^{T}(X)}{X !}\right)\right. \\
& \left.\cdot\left(\left\langle\frac{\varphi_{\Lambda}^{T}(Z)}{Z !}\right\rangle-\frac{\varphi_{\Lambda}^{T}(Z)}{Z !}\right)\right\rangle .
\end{aligned}
$$

Let $\Lambda_{d}^{\prime}$ be the region defined in Section 4, i.e.

$$
\Lambda_{d}^{\prime}=\{\xi \mid \xi \in \Lambda, d(\xi, \partial \Lambda) \leqq d\} .
$$


In (5.3) we let $\xi$ and $\zeta$ variate only in $\Lambda_{d}^{\prime}$ since all other terms are vanishing; if we take in to account that the process we are analysing is a $d$-Markov one, the mean value of the $\varphi_{\Lambda}^{T}(X)$ functions depends on those sets of $Z^{\delta}$ whose distance from $\partial \Lambda$ is less or equal to $d$ (i.e. the sets $T$ s.t. $T \cap \Lambda_{d} \neq \emptyset$, where $\Lambda_{d}$ has been defined in Definition 1 of introduction)

$$
\begin{aligned}
& =\sum_{\substack{\xi \in \Lambda_{d d}^{\prime} \\
\zeta \in \Lambda_{d}^{\prime}}} \sum_{\substack{\xi \in X \in \mathfrak{P}(\Lambda) \\
\zeta \in Z \in \mathfrak{P}(\Lambda)}} z^{|X|+|Z|} \frac{|X||Z|}{|\tilde{X}||\tilde{Z}|} \\
& \cdot\left\langle\left(\left\langle\frac{\varphi_{\Lambda}^{T}(X)}{X !}\right\rangle-\frac{\varphi_{\Lambda}^{T}(X)}{X !}\right)\left(\left\langle\frac{\varphi_{\Lambda}^{T}(Z)}{Z !}\right\rangle-\frac{\varphi_{\Lambda}^{T}(Z)}{Z !}\right)\right\rangle \\
& =\sum_{\substack{\xi \in \Lambda_{d}^{\prime} d \\
\zeta \in \Lambda^{\prime} d}} \sum_{\substack{\xi \in X \in \mathfrak{P}(\Lambda) \\
\zeta \in Z \in \mathfrak{P}(\Lambda)}} z^{|X|+|Z|} \frac{|X||Z|}{|\tilde{X}||\tilde{Z}|} \\
& \cdot\left[\left\langle\frac{\varphi_{\Lambda}^{T}(X)}{X !} \frac{\varphi_{\Lambda}^{T}(Z)}{Z !}\right\rangle-\left\langle\frac{\varphi_{\Lambda}^{T}(X)}{X !}\right\rangle\left\langle\frac{\varphi_{\Lambda}^{T}(Z)}{Z !}\right\rangle\right] \\
& =\sum_{\substack{\xi \in \Lambda_{d}^{\prime} \\
\zeta \in \Lambda_{d}^{\prime}}} \sum_{\substack{\xi \in X \in \mathfrak{P}(\Lambda) \\
\zeta \in Z \in \mathfrak{P}(\Lambda)}} z^{|X|+|Z|} \frac{|X||Z|}{|\tilde{X}||\tilde{Z}|} \\
& \cdot \sum_{Y \in \Lambda_{d d}} \sum_{\substack{Y_{1} \in \Lambda_{d} \\
Y_{2} \in \Lambda_{d}}} \frac{\varphi_{\Lambda}^{T}(X)}{X !} \frac{\varphi_{\Lambda}^{T}(Z)}{Z !}\left[f_{\Lambda_{d}}(Y)-f_{\Lambda_{d}}\left(Y_{1}\right) f_{\Lambda_{d}}\left(Y_{2}\right)\right]
\end{aligned}
$$

where the $f_{\Lambda_{d}}(Y)$ has been introduced in Lemma 2 and the computation of the expectation values has been performed on the sets $Y, Y_{1}$, and $Y_{2}$. Now by the previous observation it is possible to find two neighborhoods $\Delta_{X}$ and $\Delta_{Z}$ of $X$ and $Z$ respectively s.t. $\Delta_{X} \subset \Lambda_{d} ; \Delta_{Z} \subset \Lambda_{d}$ and the probability of $X$ depends only on $Y_{1}$ 's s.t. $Y_{1} \cap \Delta_{X} \neq \emptyset$ and the probability of $Z$ depends only on $Y_{2}$ 's s.t. $Y_{2} \cap \Delta_{Z} \neq \emptyset$. We must now distinguish between two possibilities:
a) $d\left(\Delta_{X}, \Delta_{Z}\right)>3 d$
b) $d\left(\Delta_{X}, \Delta_{Z}\right) \leqq 3 d$.
a) $d\left(\Delta_{X}, \Delta_{Z}\right)>3 d$;

we have:

(1.h.s. of (5.4))

$$
\begin{aligned}
= & \sum_{\substack{\xi \in \Lambda_{d}^{\prime} \\
\zeta \in \Lambda_{d}^{\prime}}} \sum_{\substack{\xi \in X \in \mathfrak{P}(\Lambda) \\
\zeta \in Z \in \mathfrak{P}(\Lambda)}} z^{|X|+|Z|} \frac{|X||Z|}{|\tilde{X}||\tilde{Z}|} \cdot \sum_{\substack{Y_{1} \subset \Delta_{X} \\
Y_{2} \subset \Delta_{Z}}} \frac{\varphi_{\Lambda}^{T}(X)}{X !} \frac{\varphi_{\Lambda}^{T}(Z)}{Z !} \\
& \cdot\left(f_{\Delta_{X} \cup \Delta_{Z}}\left(Y_{1} \cup Y_{2}\right)-f_{\Delta_{X}}\left(Y_{1}\right) f_{\Delta_{Z}}\left(Y_{2}\right)\right)
\end{aligned}
$$


and by Lemma 2

(1.h.s. of (5.5))

$$
\begin{aligned}
= & \sum_{\substack{\xi \in \Lambda_{d d}^{\prime} \\
\zeta \in \Lambda_{d}^{\prime}}} \sum_{\substack{\xi \in X \in \mathfrak{P}(\Lambda) \\
\zeta \in Z \in \mathfrak{P}(\Lambda)}} z^{|X|+|Z|} \frac{|X||Z|}{|\tilde{X}||\tilde{Z}|} \cdot \sum_{\substack{Y_{1} \in \Delta_{X} \\
Y_{2} \in \Delta_{Z}}} \frac{\varphi_{\Lambda}^{T}(X)}{X !} \frac{\varphi_{\Lambda}^{T}(Z)}{Z !} f_{\Delta_{X}}\left(Y_{1}\right)_{-} \\
& \cdot \mathscr{f}_{\Delta_{Z}}\left(Y_{2}\right) e^{-\alpha d\left(\Delta_{X} \Delta_{Z}\right)} \\
= & \sum_{\substack{\xi \in \Lambda_{d d}^{\prime} \\
\zeta \in \Lambda_{d}^{\prime}}} \sum_{\substack{\xi \in X \in \mathfrak{P}(\Lambda) \\
\zeta \in Z \in \mathfrak{P}(\Lambda)}} z^{|X|+|Z|} \frac{|X||Z|}{|\tilde{X}||\tilde{Z}|}\left\langle\frac{\varphi_{\Lambda}^{T}(X)}{X !}\right\rangle\left\langle\frac{\varphi_{\Lambda}^{T}(Z)}{Z !}\right\rangle e^{-\alpha d\left(\Delta_{X}, \Delta_{Z}\right)} .
\end{aligned}
$$

Choosing now $\bar{d}$ s.t. $e^{-\bar{d}}$ is "little enough" and $D_{\xi} \equiv\left\{\zeta \mid \zeta \in \Lambda_{d}^{\prime}, d(\xi, \zeta) \leqq \bar{d}\right\}$ and using (iii) of Lemma 2 and (2.5) we have

$$
\begin{aligned}
\text { (1.h.s.) of (5.6) } & \leqq \sum_{\substack{\xi \in \Lambda_{d}^{\prime} \\
\zeta \in D_{\xi}}} \sum_{\substack{\xi \in X \in \mathfrak{P}(\Lambda) \\
\zeta \in Z \in \mathfrak{P}(\Lambda)}} z^{|X|+|Z|} \frac{|X||Z|}{|\tilde{X}||\tilde{Z}|}\left\langle\left|\frac{\varphi_{\Lambda}^{T}(X)}{X !}\right|\right\rangle\left\langle\left|\frac{\varphi_{\Lambda}^{T}(Z)}{Z !}\right|\right\rangle e^{-\alpha \bar{d}} \\
& =M_{5} \cdot|\Lambda| O\left(\varepsilon_{d}(\Lambda)\right)
\end{aligned}
$$

where $M_{5}$ is a suitable function of $(z,\|\Phi\|)$.

b) if $d\left(\Delta_{X}, \Delta_{Z}\right) \leqq 3 d$ we have, putting $D_{\xi}^{d} \equiv\left\{\zeta \mid \zeta \in \Lambda_{d}^{\prime}, d(\zeta, \zeta) \leqq 3 d\right\}$,

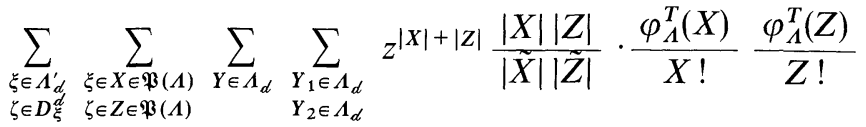

$$
\begin{aligned}
& \cdot\left[f_{\Lambda_{d}}(Y)-f_{\Lambda_{d}}\left(Y_{1}\right) f_{\Lambda_{d}}\left(Y_{2}\right)\right]= \\
& \leqq \sum_{\substack{\xi \in \Lambda_{d}^{\prime} \\
\zeta \subset D \bar{\xi}^{\prime}}} \sum_{Y \in \Lambda_{d}}\left[\sum_{\substack{\xi \in X \in \mathfrak{P}(\Lambda) \\
\zeta \in Z \in \mathfrak{P}(\Lambda)}} z^{|X|+|Z|} \frac{|X||Z|}{|\tilde{X}||\tilde{Z}|}\left|\frac{\varphi_{\Lambda}^{T}(X)}{X !}\right|\left|\frac{\varphi_{\Lambda}^{T}(Z)}{Z !}\right|\right] f_{\Lambda_{d}}(Y) \\
& +\sum_{\substack{\xi \in \Lambda_{d}^{\prime} \\
\zeta \zeta D_{\xi}}} \sum_{\substack{Y_{1} \in \Lambda_{d} \in \Lambda_{d} \\
Y_{2}}}\left[\sum_{\substack{\xi \in X \in \mathfrak{P}(\Lambda) \\
\zeta \in Z \in \mathfrak{P}(\Lambda)}} Z^{|X|+|Z|} \frac{|X||Z|}{|\tilde{X}||\tilde{Z}|}\left|\frac{\varphi_{\Lambda}^{T}(X)}{X !}\right|\left|\frac{\varphi_{\Lambda}^{T}(Z)}{Z !}\right|\right] f_{\Lambda_{d}}\left(Y_{1}\right) f_{\Lambda_{d}}\left(Y_{2}\right) .
\end{aligned}
$$

Using the same approximations as in (4.4), (4.7), we find ever for this case b)

$$
\left\langle\left(\delta\langle k\rangle_{Y}\right)^{2}\right\rangle \simeq|\Lambda| M_{6} \cdot O\left(\varepsilon_{d}(\Lambda)\right) .
$$

\section{Concluding Remarks}

1) The above technique is very general. Therefore its results should be far from the best for specific choices of $\Phi$ (e.g. if $\delta=1$ the local central limit theorem should hold for all the Gibbs processes with finite range [5]).

However the best results obtainaible with the technique of this paper should be quite near to the best possible that can be obtained with the same degree of generality.

Of course the results of this paper seem far from being the best possible the technique can provide. 
2) A great improvement of our results could be obtained if one could a priori be sure that

$$
\begin{gathered}
\sum_{0 \in X \in \mathfrak{P}(\Lambda)} z^{|X|} \frac{\varphi^{\mathrm{T}}(X)}{X !}(1-\cos t|X|)>0 \quad 0 \leqq t \leqq \pi \\
\sum_{\substack{0 \in X \\
X \in \mathfrak{P}(\Lambda)}} z^{|X|} \frac{\varphi^{T}(X)}{X !}|X|>0
\end{gathered}
$$

since in this case the conditions for the validity of Theorems 2, 3 would simply reduce to the condition for the validity of Lemma 1, i.e. simply z $C\left(\Phi^{\prime}\right)<1$.

Another improvement that it would be interesting to obtain is the extension of Theorems 2, 3 to a region of $\left(\mu, \Phi^{\prime}\right)$ of the form

$$
\frac{z}{1+z} O(\|\Phi\|)<1 \text {. }
$$

The reason why it is possible to hope for such a generalization is because a number of results are known for $Z(\Lambda, 1)$ on a region of the form (6.3) [10].

3) It is known that a process $\mu$ is ergodic (hence the fluctuations of $k-\langle k\rangle$ are $O(A))$ if $\mu$ is the only translationally invariant Gibbs process having the conditional probabilities $(1.1)[1,4]$. It is nuclear wether one also expects the local central limit theorem to hold under this sole assumption: the answer is probably no, but no-example is known.

4) One can define, as mentioned in Section 1, Gibbs processes with a potential $\Phi(X)$ which does not vanish for $\operatorname{diam} X$ large enough.

The technique of this paper extends to cover some of these processes: the error term will of course become larger and connected both only with the shape of $\Lambda$ and the mode of approach to zero of $\Phi(X)$ as $\operatorname{diam} X \rightarrow \infty$; the theorem will probably fail for too slow rates.

5) There are several $\Phi$ 's for which one can prove that there is just one Gibbs process associated with the conditional probabilities (1.1). These cases provide a number of processes for which a local central limit theorem is expected to hold but is still umproved. Trying to solve this problem for these particular $\Phi$ 's would probably teach something of interest.

We give just one "classic" example [11]

$$
\begin{array}{rlll}
\Phi^{\prime}(X) \equiv 0 & \text { if } & |X|>2 \\
\Phi^{\prime}(X, Y)=-J & \text { if } & |X-Y|=1 \quad \mu \neq-2 J . \\
=0 & \text { if } & |X-Y| \neq 1 .
\end{array}
$$

The above $\Phi$ 's give rise to Markov processes known in Physics as Gibbs states of an Ising model in non zero field. If $2 J$ is large enough 
it is known there are at least two extremal translation invariant processes with the same conditional probabilities [12]; if $J$ is still large it is known that there are only two extremal states [13].

\section{Appendix A: Proof of Lemma 1}

Let $\mathfrak{F}_{A}$ be the space of the functions on $\mathfrak{P}(\Lambda)$. We call

$$
\mathfrak{F}_{\Lambda}^{0} \equiv\left\{\varphi \mid \varphi \in \mathfrak{F}_{\Lambda}, \varphi(\emptyset)=0\right\} ; \quad \mathfrak{F}_{\Lambda}^{1} \equiv\left\{\varphi \mid \varphi \in \mathfrak{F}_{\Lambda}, \varphi(\emptyset)=1\right\} .
$$

The symbol 1 will denote the element $1 \in \mathfrak{F}_{A}$ such that $1(\emptyset)=1, \underline{1}(X) \equiv 0$ if $|X|>1$. Clearly $\mathfrak{F}_{A}^{1}=\left\{\varphi^{\prime} \mid \varphi^{\prime} \in \mathfrak{F}_{A}, \varphi^{\prime}=1+\varphi, \varphi \in \mathfrak{F}_{A}^{0}\right\}$.

We define on $\mathfrak{F}_{A}$ a commutative convolution ${ }^{4}$

$$
\left(\varphi_{1} \cdot \varphi_{2}\right)(X)=\sum_{X_{1} \cup X_{2}=X} \varphi_{1}\left(X_{1}\right) \varphi_{2}\left(X_{2}\right) \frac{X !}{X_{1} ! X_{2} !}
$$

where the sum is over all the possible decompositions of $X$ into an ordered pair of subsets.

Define for $\varphi \in \mathfrak{F}_{A}^{0}$

$$
\operatorname{Exp} \varphi=\sum_{n=0}^{\infty} \frac{\varphi^{n}}{n !}
$$

where $\varphi^{0}=1$ and $\varphi^{n}=\varphi \cdot \varphi \cdot \varphi \ldots$ ( $n$ times); (A.2) is to be understood as $(\operatorname{Exp} \varphi)(X)=\sum_{n=0} \frac{\varphi^{n}(X)}{n !} ; X \in \mathfrak{P}(\Lambda)$ and the series involves only a finite number of terms if $\varphi \in \mathfrak{F}_{\Lambda}^{0}$. We write also with the same meaning as for (A.2):

$$
\log (1+\varphi)=\sum_{n=1}^{\infty} \frac{(-1)^{n+1}}{n} \varphi^{n}
$$

The operator $D_{X}$ is defined on $\mathfrak{F}_{A}$ as

$$
\left(D_{X} \varphi\right)(Y)=\varphi(X \cup Y) \quad X \in \mathfrak{P}(\Lambda)
$$

where the union $X \cup Y$ takes, of course, the multiplicities into account.

We find

$$
\begin{gathered}
D_{\xi}\left(\varphi_{1} \cdot \varphi_{2}\right)=\left(D_{\xi} \varphi_{1}\right) \cdot \varphi_{2}+\varphi_{1}\left(D_{\xi} \varphi_{2}\right) \\
D_{\xi}(\operatorname{Exp} \varphi)=D_{\xi}(\varphi) \cdot \operatorname{Exp} \varphi \quad \forall \varphi \in \mathfrak{F}_{\Lambda}^{0} .
\end{gathered}
$$

${ }^{4}$ It is at this point that there is an error in Section 6 of [9]. The error is copied in the appendix of the paper of Ref. [14] below (with no consequences for the results) and is corrected with notations in Gallavotti: Commun. math. Phys. 27, 103 (1972) and with better notations in the second paper of Ref. [14]. A corrent theory of the algebra over $\mathfrak{F}_{A}$ has been developed also by Shen: J. Math. Physics 5, 754 (1972). 
Furthermore if $\chi(\xi)$ is a complex function on $Z^{\delta}$ and $\chi(X)=\prod_{\xi \in X}[\chi(\xi)]^{n_{\xi}}$ if $X=\left\{n_{\xi}\right\}$ and if $\varphi \in \mathfrak{F}_{A}^{1}$ and $\varphi^{T}=\log \varphi$ are such that

then

$$
\sum_{X \in \mathfrak{P}(\Lambda)} \frac{|\varphi(X)|}{X !}|\chi(X)|<\infty
$$

$$
\begin{gathered}
\sum_{X \in \mathfrak{P}(\Lambda)} \frac{\left|\varphi^{T}(X)\right|}{X !}|\chi(X)|<\infty \\
\sum_{X \in \mathfrak{P}(\Lambda)} \frac{\varphi(X)}{X !} \chi(X)=\exp \sum_{X \in \mathfrak{P}(\Lambda)} \frac{\varphi^{T}(X)}{X !} \chi(X) .
\end{gathered}
$$

This formula follows from the easily checked formal relation:

$$
\sum_{X \in \mathfrak{P}(\Lambda)} \frac{\left(\varphi_{1} \cdot \varphi_{2}\right)(X)}{X !} \chi(X)=\left[\sum_{X \in \mathfrak{P}(\Lambda)} \frac{\varphi_{1}(X)}{X !} \chi(X)\right]\left[\sum_{X \in \mathfrak{P}(\Lambda)} \frac{\varphi_{2}(X)}{X !} \chi(X)\right] .
$$

To prove the lemma we are thus naturally led to consider $\varphi_{A} \in \mathfrak{F}_{A}^{1}$ [see (3.1)]:

$$
\varphi_{\Lambda}(X)=\left\{\begin{array}{lll}
e^{-U(X)-I(X)} & \text { if } & X !=1 \\
0 & \text { if } & X !>1
\end{array}\right.
$$

$\varphi_{\Lambda}(\phi)$ has to be interpreted as 1 .

We shall also consider $\varphi_{A}^{T}(X)=z^{|X|} \lambda(X) \varphi_{A}^{T}(X)$.

Clearly if $\varphi_{A}^{T}=\log \varphi_{A}$ then $\varphi_{A}^{\prime T}(X)=z^{|X|} \lambda(X) \varphi_{A}^{T}(X)$.

Therefore in order to apply (A.8) to deduce statement i) of Lemma 1 we reed only to prove (A.7), for $\varphi_{A}$ with $\chi(X)=z^{|X|} \lambda(X)$. Since the first of the two relations (A.7) is trivially true we need only prove the second.

For this purpose consider the functions:

$$
\Delta_{X}(P)=\left(\varphi_{\Lambda}^{\prime-1} \cdot D_{X} \varphi_{\Lambda}\right)(P) \quad P \in \mathfrak{P}(\Lambda)
$$

for $X !=1, X \neq \emptyset$; here $\varphi_{A}^{\prime-1}$ is defined by the equation $\varphi_{A}^{\prime-1} \cdot \varphi_{A}=1$ (which can be solved easily by recursion since $\varphi_{A} \in \mathfrak{F}_{A}^{1}$ ).

The interst of the above functions lies in the fact that, using (A.6)

$$
\begin{aligned}
\Delta_{\xi}(P) & =\left(\varphi_{A}^{\prime-1} \cdot D_{\xi} \varphi_{\Lambda}^{\prime}\right)(P)=\left(\varphi_{A}^{\prime-1} \cdot D_{\xi} \varphi_{A}^{\prime T}\right)(P) \\
& =\left(\varphi_{A}^{\prime-1} \cdot\left(D_{\xi} \varphi_{A}^{\prime T}\right) \cdot \operatorname{Exp} \varphi_{A}^{\prime T}\right)(P) \\
& =\left(\varphi_{A}^{\prime-1} \cdot\left(D_{\xi} \varphi_{A}^{\prime T}\right) \varphi_{A}^{\prime}\right)(P) \\
& =\left(D_{\xi} \varphi_{A}^{\prime T}\right)(P)=\varphi_{A}^{\prime T}(\xi \cup P) .
\end{aligned}
$$

We can easily write an equation for $\Delta_{X}(P)$, in fact by definition:

$$
\frac{\Delta_{X}(P)}{P !}=\sum_{P_{2} \subset P} \frac{\varphi_{\Lambda}^{\prime-1}\left(P / P_{2}\right)}{\left(P / P_{2}\right) !} \cdot \frac{\varphi_{\Lambda}^{\prime}\left(P_{2} \cup X\right)}{P_{2} !}
$$


notice that we can and shall assume $P_{2} ! \equiv 1$ i.e. $P_{2}=\tilde{P}_{2} \subset \tilde{P}$ because of the definition (A.10) of $\varphi_{A}$ and furthermore we can assume $P_{2} \cap X=\emptyset$. Hence

$$
\begin{gathered}
\frac{\Delta_{X}(P)}{P !}=\sum_{\substack{P_{2} \subset \tilde{P} \\
P_{2} \cap X=\emptyset}} \frac{\varphi_{\Lambda}^{\prime-1}\left(P / P_{2}\right)}{\left(P / P_{2}\right) !} \frac{\varphi_{\Lambda}^{\prime}\left(X \cup P_{2}\right)}{1 !} \\
=z \lambda\left(\xi_{1}\right) e^{-U_{1}(X)} \sum_{\substack{P_{2} \subset P \\
P_{2} \cap X=\emptyset}} \frac{\varphi_{\Lambda}^{\prime-1}\left(P / P_{2}\right)}{P / P_{2} !} z^{\left|X^{(1)} \cup P_{2}\right|} \cdot \lambda\left(X^{(1)} \cup P_{2}\right) \\
\cdot e^{-U\left(X^{(1)} \cup P_{2}\right)-I\left(X^{(1)} \cup P_{2}\right)} \cdot e^{-\sum_{R \subset P_{2} \cap Y}^{\sum} W_{1}(X, R)}
\end{gathered}
$$

where $\xi_{1}$ is a point $\xi_{1} \in X ; X^{(1)}=X /\left\{\xi_{1}\right\}, Y$ is the set outside $\Lambda$ (fixed at the beginning) and

$$
\begin{aligned}
U_{1}(X) & =\sum_{\xi_{1} \in Q \subset X} \Phi^{\prime}(Q) \\
W_{1}(X, R) & =\sum_{\xi_{1} \in T \subset X} \Phi^{\prime}(T \cup R) .
\end{aligned}
$$

Now write (for $R \cap X \neq \emptyset$ ):

$$
e^{-\underset{R \subset P_{2} \cap Y}{\sum} W_{1}(X, R)}=\prod_{R \subset P_{2} \cup Y}\left(e^{-W_{1}(X, R)}-1+1\right)=\sum_{S \subset P_{2} \cup Y} K_{1}(X, S)
$$

with $K_{1}(X, \emptyset)=1$ and

$$
K_{1}(X, S)=\sum_{n \geqq 1} \sum_{\substack{R_{1} \ldots R_{n} \\ \cup R_{i}=S}} \prod_{i=1}^{n}\left(e^{-W_{1}\left(X, R_{t}\right)}-1\right)
$$

where the sum runs over all the coverings of $S$ (see [8], p. 276): we find, taking into account the definition (A.10) of $\varphi_{\Lambda}$ (putting $P_{2}=P^{\prime} \cup(S \cap \tilde{P})$ ),

$$
\begin{aligned}
\frac{\Delta_{X}(P)}{P !}= & z \lambda\left(\xi_{1}\right) e^{-U_{1}(X)} \sum_{\substack{P_{2} \subset \tilde{P} \\
P_{2} \cap \xi_{1}=\emptyset}} \sum_{\substack{S \subset P_{2} \cup Y \\
S \cap X=\emptyset}} K_{1}(X, S) \\
& \cdot \varphi_{A}^{\prime}\left(X^{(1)} \cup P_{2}\right) \cdot \frac{\varphi_{A}^{\prime-1}\left(P / P_{2}\right)}{\left(P / P_{2}\right) !} \\
= & z \lambda\left(\xi_{1}\right) e^{-U_{1}(X)} \sum_{\substack{S \subset \tilde{P} \cup Y \\
\tilde{P}_{1} \cup Y}} K_{1}(X, S) \sum_{T \subset \tilde{P} \cap \xi_{1}}(-1)^{|T|} \\
& \cdot \sum_{P^{\prime \prime} \subset(\widetilde{P} \mid S \cup T)} \frac{\varphi_{A}^{\prime-1}\left(P / P^{\prime \prime} \cup S \cup T\right)}{\left(P / P^{\prime \prime} \cup S \cup T\right) !} \\
& \cdot \varphi_{\Lambda}^{\prime}\left(X^{(1)} \cup(S \cap \tilde{P}) \cup T \cup P^{\prime \prime}\right) .
\end{aligned}
$$


Since in (A.20) $\sum_{P^{\prime \prime} \subset \tilde{P} / S \cup T}=\sum_{P^{\prime \prime} \subset \mathcal{P} / S \cup T}$, again as consequence of the definition of $\varphi_{\Lambda}$, and since

$$
\left(P / P^{\prime} \cup S \cup T\right)=\left(P / P^{\prime} \cup(S \cap \tilde{P}) \cup T\right)
$$

it follows

$$
\begin{aligned}
\frac{\Delta_{X}(P)}{P !}= & z \lambda\left(\xi_{1}\right) e^{-U_{1}(X)} \sum_{\substack{S \subset \tilde{P} \cup Y \\
S \cap X=\emptyset}} K_{1}(X, S) \sum_{T \subset P \cap \xi_{1}}(-1)^{T} \\
& \cdot \Delta_{X^{1} \cup(S \cap \tilde{P}) \cup T}(P / T \cup(S \cap \tilde{P})) /(P / T \cup(S \cap \tilde{P})) !
\end{aligned}
$$

in (A.21) appear terms of the type $\Delta_{\Phi}(Q)$ : these terms are not defined, however, if one keeps track of them in the definition, one finds that $\Delta_{\Phi}(Q)$ has to be interpreted as $1(Q)$.

Put now

$$
I_{n}=\sup _{\substack{h+k=n \\ h \geqq 1 \\ k \geqq 0}} \sup _{\substack{X \in \mathfrak{P}(\Lambda) \\|X|=h}} \sum_{\substack{P \subset \mathfrak{P}(\Lambda) \\|P|=k}} \frac{\left|\Delta_{X}(P)\right|}{P !} .
$$

Using (A.21) and $|\lambda| \leqq 1$ and choosing $\xi_{1}=\xi$ and $|P|=k,|X|=h$

$$
\begin{aligned}
& \sum_{\substack{P \\
|P|=k}} \frac{\left|\Delta_{\xi \cup X}(P)\right|}{P !} \\
& \leqq z e^{-U(X \cup \xi)} \sum_{\substack{P \\
|P|=k}} \sum_{S \subset \tilde{P} \cup Y} K_{1}(X \cup \xi, S) \cdot \sum_{T \subset \tilde{P} \cup \xi} \frac{\left|\Delta_{X \cup(S \cap \tilde{P})}(P / T \cup(S \cap \tilde{P}))\right|}{(P / T \cup(S \cap \tilde{P})) !} \\
& \leqq I_{n} z e^{-U_{1}(X \cup \xi)} \sum_{\substack{S \subset A \cup Y \\
S \cap(X \cup \xi)=\emptyset}}\left|K_{1}(X \cup \xi, S)\right| .
\end{aligned}
$$

The coefficient of $I_{n}$ in (A.23) has been estimated in Ref. [8], p. 276 and using that estimate and taking the supremum on the 1.h.s. of (A.23) one finds [see (2.4)]:

$$
I_{n+1} \leqq I_{n} z C(\Phi) .
$$

Since $I_{1}=\left|\left(\varphi_{A}^{\prime-1} \cdot D_{\xi} \varphi_{\Lambda}^{\prime}\right)(\phi)\right|=\left|\varphi_{\Lambda}^{\prime-1}(\phi) \cdot \varphi_{\Lambda}(\xi)\right|=z$ we find

$$
I_{n+1} \leqq z(z C(\Phi))^{n}
$$

Formula (3.4) follows by observing that $\varphi_{A}^{\prime T}(X)=z^{|X|} \lambda(X) \varphi_{A}^{T}(X)$ and using (A.12).

The independence and translation invariance of $\varphi_{\Lambda}^{T}(X)$ 's on $\Lambda$ when $d(X, \partial \Lambda)>d$ follow from the definition:

$$
\begin{aligned}
\varphi_{\Lambda}^{T}(X) & =\left[\log \varphi_{\Lambda}(X)\right] \\
& =\sum_{n \geqq 1} \frac{(-1)^{n+1}}{n} \sum_{X_{1} \cup X_{2} \cup \cdots \cup X_{n}=X}\left[\prod_{i} \frac{\varphi_{\Lambda}\left(X_{i}\right)}{X_{i} !}\right] X !
\end{aligned}
$$


and from the fact that $\varphi_{A}(X)$ 's are independent and translation invariant (actually equal to $e^{-U(X)}$ or zero) if $d(X, \partial \Lambda)>d$. Formulas (3.5), (3.6) follow from the remark that $\varphi_{\Lambda}^{T}(X)=0$ if $\operatorname{diam} X>|\tilde{X}| d$. For details see [14].

\section{Appendix B}

In this appendix we shall prove the statement formulated in Lemma 2. Let us first remark that it is possible to express the $f(Y)$ 's, introduced in Lemma 2, trough the $\varphi_{\Lambda}^{T}(Y)$ 's as follows: let $M$ a finite region of $Z^{\delta}$ and $Y \subset \Lambda \subset M, X \subset M / \Lambda$; we get

$$
\begin{aligned}
f_{\Lambda}(Y) & =\lim _{|M| \rightarrow \infty} \sum_{X \subset M / \Lambda} f_{M}(X \cup Y)=\lim _{|M| \rightarrow \infty} \frac{\sum_{X \subset M / A} e^{-U(X)-U(Y)-I(X, Y)}}{\sum_{X \subset M} e^{-U(X)}} \\
& =\lim _{|M| \rightarrow \infty} e^{-U(Y)} \frac{\sum_{X \subset M / \Lambda} \varphi_{M, Y}(X)}{\sum_{X \subset M} \varphi_{M}(X)}
\end{aligned}
$$

where the notation $\varphi_{M, Y}(X)$ emphasizes the $Y$-dependence of those $\varphi_{M}(X)$ 's which appear in the numerator. Using Lemma 1, (i) we obtain

$$
\begin{aligned}
f_{\Lambda}(Y)= & \lim _{|M| \rightarrow \infty} e^{-U(Y)} \frac{\exp \sum_{X \in \mathfrak{P}(M / \Lambda)} \varphi_{M, Y}^{T}(X) / X !}{\exp \sum_{X \in \mathfrak{P}(M)} \varphi_{M}^{T}(X) / X !} \\
= & \lim _{|M| \rightarrow \infty} e^{-U(Y)} \exp \left\{-\sum_{X \in \mathfrak{P}(\Lambda)} \frac{\varphi_{M}^{T}(X)}{X !}-\sum_{\substack{X \notin \mathfrak{P}(\Lambda) \\
X \cap A=\emptyset}} \frac{\varphi_{M}^{T}(X)}{X !}\right. \\
& +\sum_{X \in \mathfrak{P}(\boldsymbol{M} / \boldsymbol{A})} \frac{1}{X !}\left[\varphi_{M, Y}^{T}(X)-\varphi_{M}^{T}(X)\right] .
\end{aligned}
$$

In order to demonstrate the (3.9) formula we need to calculate $f_{\Lambda_{1} \cup \Lambda_{2}}\left(Y_{1} \cup Y_{2}\right)$ where $\Lambda_{1} \subset M, \Lambda_{2} \subset M, Y_{1} \subset \Lambda_{1}, Y_{2} \subset \Lambda_{2}$.

The (B.2) formula yields

$$
\begin{aligned}
& f_{\Lambda_{1} \cup \Lambda_{2}}\left(Y_{1} \cup Y_{2}\right) \\
& =e^{-U\left(Y_{1} \cup Y_{2}\right)} \lim _{|M| \rightarrow \infty} \exp \left\{-\sum_{X \in \mathfrak{P}\left(\Lambda_{1} \cup \Lambda_{2}\right)} \frac{\varphi_{M}^{T}(X)}{X !}-\sum_{\substack{X \notin \mathfrak{P}\left(\Lambda_{1} \cup \Lambda_{2}\right) \\
\left(X \cap\left(\Lambda_{1} \cup \Lambda_{2}\right)\right)=\emptyset}} \frac{\varphi_{M}^{T}(X)}{X !}\right. \\
& +\sum_{X \in \mathfrak{P}\left(M / \Lambda_{1} \cup \Lambda_{2}\right)}(1 / X !)\left[\varphi_{M, Y_{1} \cup Y_{2}}^{T}(X)-\varphi_{M}^{T}(X)\right] .
\end{aligned}
$$

Since $d\left(\Lambda_{1}, \Lambda_{2}\right)>3 d$, in the hypothesis of Lemma 2, from the Definition 3.2 it follows $I\left(Y_{1}, Y_{2}\right)=0$; therefore reordering the summations in 
(B.3) and having into account obvious sets properties we have

$$
\begin{aligned}
& f_{\Lambda_{1} \cup A_{2}}\left(Y_{1} \cup Y_{2}\right) \\
& =e^{-U\left(Y_{1}\right)-U\left(Y_{2}\right)} \lim _{|M| \rightarrow \infty} \exp \left\{-\sum_{X \in \mathfrak{P}\left(\Lambda_{1}\right)} \frac{\varphi_{M}^{T}(X)}{X !}-\sum_{X \in \mathfrak{P}\left(\Lambda_{2}\right)} \frac{\varphi_{M}^{T}(X)}{X !}\right. \\
& +-\sum_{\substack{X \in \mathfrak{P}\left(\Lambda_{1} \cup \Lambda_{2}\right) \\
X \notin \mathfrak{P}\left(\Lambda_{1}\right) \\
X \notin \mathfrak{P}\left(\Lambda_{2}\right)}} \frac{\varphi_{M}^{T}(X)}{X !}+\sum_{\substack{X \in \mathfrak{P}\left(M / \Lambda_{1}\right) \\
X \cap \Lambda_{1} \neq \emptyset}}(1 / X !)\left[\varphi_{M, Y_{1}}^{T}(X)-\varphi_{M}^{T}(X)\right] \\
& +\sum_{\substack{X \in \mathfrak{P}\left(M / \Lambda_{1}\right) \\
X \cap \Lambda_{1_{d}} \neq \emptyset \\
X \cap\left(\Lambda_{2} \cup \Lambda_{2_{d}}\right) \neq \emptyset}}(1 / X !)\left[\varphi_{M, Y_{1}}^{T}(X)-\varphi_{M}^{T}(X)\right] \\
& +\sum_{\substack{X \in \mathfrak{P}\left(M / \Lambda_{2}\right) \\
X \cap \Lambda_{2}}}(1 / X !)\left[\varphi_{M, Y_{2}}^{T}(X)-\varphi_{M}^{T}(X)\right] \\
& X \cap \Lambda_{2} \neq \emptyset \\
& +-\sum_{\substack{X \in \mathfrak{P} M / \boldsymbol{A}_{2} \\
X \cap \boldsymbol{A}_{d_{d}} \neq \emptyset}}(1 / X !)\left[\varphi_{M, Y_{2}}^{T}(X)-\varphi_{M}^{T}(X)\right] \\
& \left.+\sum_{\substack{X \in \mathfrak{P}\left(M / \Lambda_{1} \cup \Lambda_{2}\right) \\
X \cap \Lambda_{1} \neq \emptyset \\
X \cap \Lambda_{2_{d}} \neq \emptyset}}^{X \cap\left(\Lambda_{d} \cup \Lambda_{1_{d}}\right) \neq \emptyset}(1 / X !)\left[\varphi_{M, Y_{1} \cup Y_{2}}^{T}(X)-\varphi_{M}^{T}(X)\right]\right\}
\end{aligned}
$$

where $\Lambda_{i_{d}} \equiv\left\{\xi \mid \xi \in \Lambda_{i}, d\left(\xi, \partial \Lambda_{i}\right) \leqq d\right\} i=1,2$, and the hypothesis $d\left(\Lambda_{1}, \Lambda_{2}\right)>3 d$ has conceeded to put $\varphi_{M, Y_{1} \cup Y_{2}}^{T}(X)=\varphi_{M, Y_{1}}^{T}(X)$ [resp. $\left.\varphi_{M, Y_{1} \cup Y_{2}}^{T}(X)=\varphi_{M, Y_{2}}^{T}(X)\right]$ whenever the summation was only extended over $X \in \mathfrak{P}\left(\Lambda_{1}\right)$ [resp. $\left.X \in \mathfrak{P}\left(\Lambda_{2}\right)\right]$.

Let now consider the difference $\mathscr{f}_{\Lambda_{1} \cup \Lambda_{2}}\left(Y_{1} \cup Y_{2}\right)-f_{\Lambda_{1}}\left(Y_{1}\right) f_{\Lambda_{2}}\left(Y_{2}\right) \mid$; from (B.4) formula we get

$$
\begin{aligned}
& f_{\Lambda_{1} \cup \Lambda_{2}}\left(Y_{1} \cup Y_{2}\right)-f_{\Lambda_{1}}\left(Y_{1}\right) f_{\Lambda_{2}}\left(Y_{2}\right) \mid \\
& \leqq \lim _{|M| \rightarrow \infty} f_{\Lambda_{1}}\left(Y_{1}\right) f_{\Lambda_{2}}\left(Y_{2}\right) \exp \left\{-\sum_{\substack{X \in \mathfrak{P}\left(\Lambda_{1} \cup \Lambda_{2}\right) \\
X \notin \mathfrak{P}\left(\Lambda_{1}\right) \\
X \notin \mathfrak{P}\left(\Lambda_{2}\right)}} \frac{\left|\varphi_{M}^{T}(X)\right|}{X !}\right. \\
& +\sum_{\substack{X \notin \mathfrak{P}\left(\Lambda_{1} \cup \Lambda_{2}\right) \\
X \cap \Lambda_{1} \neq \theta \\
X \cap 0}} \frac{\left|\varphi_{M}^{T}(X)\right|}{X !}-\sum_{\substack{X \in \mathfrak{P}\left(\boldsymbol{M} / \Lambda_{1}\right) \\
X \cap \Lambda_{1} \neq \emptyset \\
X \cap \Lambda_{2} \neq \emptyset}}(1 / X !)\left[\varphi_{M, Y_{1}}^{T}(X)-\varphi_{M}^{T}(X)\right] \\
& +-\sum_{X \in \mathfrak{P}\left(M / \Lambda_{2}\right)}(1 / X !)\left[\varphi_{M, Y_{2}}^{T}(X)-\varphi_{M}^{T}(X)\right] \\
& X \cap \Lambda_{2} \neq \emptyset \\
& X \cap\left(\Lambda_{2} \cup \Lambda_{2}\right) \neq \emptyset \\
& +\sum_{\substack{X \in \mathfrak{P}\left(M / \Lambda_{1} \cup \Lambda_{2}\right) \\
X \cap \Lambda_{1} \neq \emptyset \\
X \cap \Lambda_{2} \neq \emptyset}}(1 / X !)\left|\varphi_{M, Y_{1} \cup Y_{2}}^{T}(X)-\varphi_{M}^{T}(X)\right| .
\end{aligned}
$$


If iv) of Lemma 1 is taken into account and the fact that $\varphi_{M, Y_{1} \cup Y_{2}}^{T}(X)$ 's satisfy the same properties as $\varphi_{M, Y_{1}}^{T}(X)^{\prime}$ 's do, $i=1,2$ we obtain

$$
f_{\Lambda_{1} \cup \Lambda_{2}}\left(Y_{1} \cup Y_{2}\right)-f_{\Lambda_{1}}\left(Y_{1}\right) f_{\Lambda_{2}}\left(Y_{2}\right) \mid \leqq f_{\Lambda_{1}}\left(Y_{1}\right) f_{\Lambda_{2}}\left(Y_{2}\right) e^{-\alpha d\left(\Lambda_{1}, \Lambda_{2}\right)}
$$

where $\alpha=\alpha(Z,\|\Phi\|)$ is a suitable constant.

\title{
References
}

1. Dobrushin, R. L.: Theory Prob. Appl. 13, 197 (1968); see also Dobrushin, R. L.: Theory Prob. Appl. 15, 458 (1970) and appended references

2. Spitzer, F.: Am. Math. Monthly 78, 142 (1971) Averintzev, M. B.: Prob. Per. Inform. 6, 100 (1970)

3. Griffiths, R.B., Ruelle, D.: Commun. math. Phys. 23, 169 (1971)

4. Lanford, O., Ruelle, D.: Commun. math. Phys. 13, 194 (1969)

5. Kolmogorov, A. N.: In Selected translations in probability theory, Vol. 2, p. 109. Providence, R.I.: AMS and IMS

6. Ruelle, D.: Statistical mechanics, p. 83; New York: Benjamin 1969

7. Ryauba,B.: Litovsk, Math. Sb. 2, 193 (1962); 3, 207 (1963)

8. Gallavotti, G., Miracle-Sole,S.: Commun. math. Phys. 7, 274 (1968)

9. Fisz, M.: Probability theory and matematical statistic. London: John Wiley \& Sons 1963

10. Ruelle,D.: See [6], Chapter 5

11. Ruelle,D.: See [6], Chapter 5

12. Ruelle,D.: See [6], p. 87

13. Gallavotti, G., Miracle-Sole,S.: Phys. Rev. 5B, 2555 (1972)

14. Gallavotti,G., Martin-Löf,A.: Commun. math. Phys. 25, 87 (1972) Appendix 1. This appendix is affected by combinatorial errors (though results are correct). A correct version containing also strong results can be found in the appendix to the paper by Gallavotti,G., Martin-Löf,A., Miracle-Sole,S.: To appear in the "Lecture notes of the Battelle Summer Rencontres in Mathematics and Physics, 1971". Lecture Notes in Physics. Berlin-Heidelberg-New York: Springer

15. Diliberto,F., Gallavotti,G., Russo,F.: Markov processes, Bernoulli shifts, Ising model, to appear in Commun. math. Phys.

16. Halfina: Math. Sbornik T. 80 (122), $\mathrm{n}^{0} 1$ (9), pp. 3-51

17. Milnos, Halfina: Izvetija A.N. Ser. Math. T. 34.5, pp. 1173-1191 (1970)

Communicated by G. Gallavotti

\author{
G. Del Grosso \\ Istituto Matematico \\ Università di Roma \\ Via Vicenza 23 \\ I-00185 Roma, Italy
}

